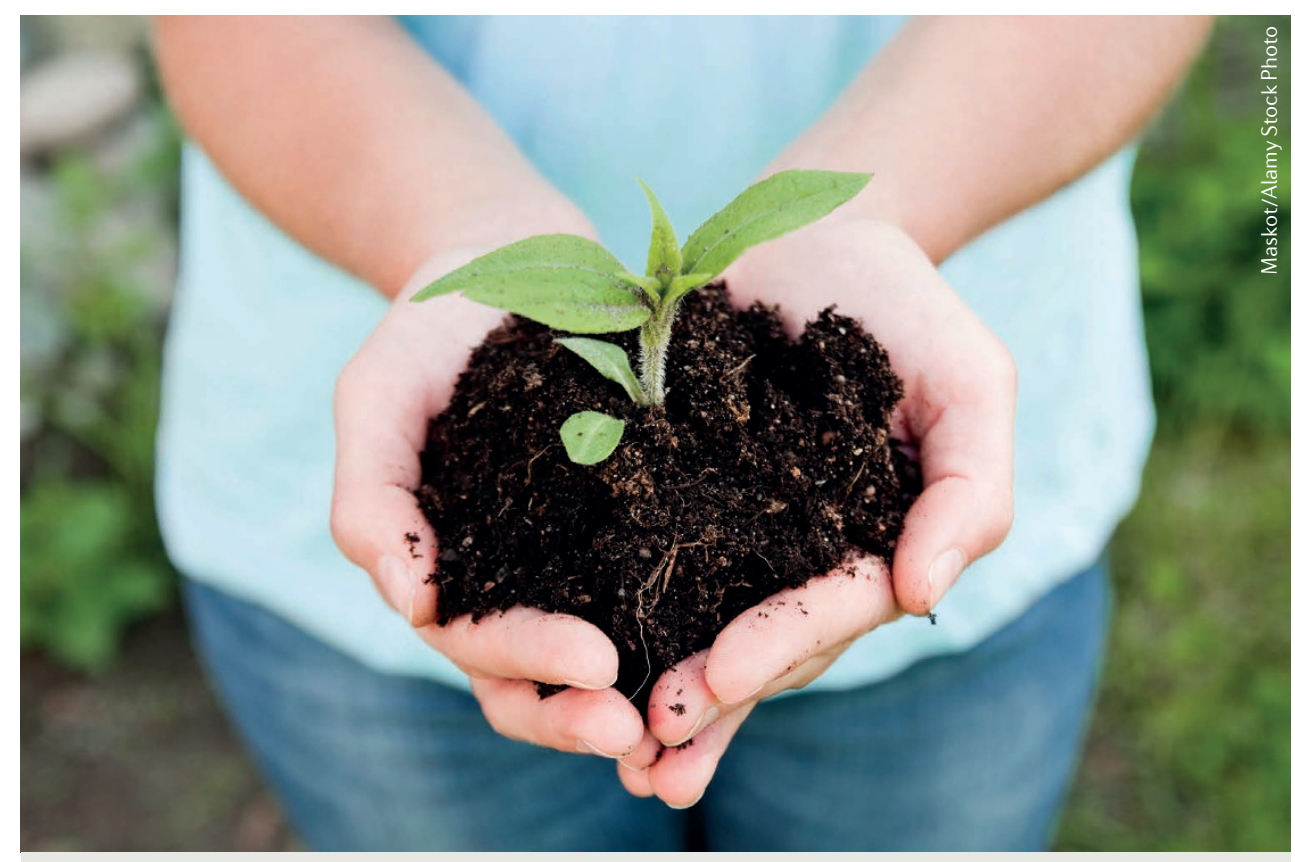

CANCER

\title{
Fibroblast subtype provides niche for cancer stem cells
}

The role of cancer-associated fibroblasts (CAFs) in tumour formation has been controversial. A new paper in Cell demonstrates that a subset of CAFs, defined by the presence of the cell surface markers CD10 and GPR77, promotes tumour formation and chemoresistance by providing a niche for cancer stem cells (CSCs). Targeting these cells with antibodies against GPR77 reduced tumour formation and restored chemosensitivity in animal models.

In the past, "strategies targeting CAFs were greatly hampered by the heterogeneity of the fibroblasts," says corresponding author and breast surgeon Erwei Song. "Some cancer patients fail to respond to multiple chemotherapeutic regimens, and the CAF subsets in chemosensitive and chemoresistant patients are different," he explains. His group therefore set out to define those differences, starting by examining the mRNA profiles of CAFs isolated from chemosensitive or chemoresistant tumours. They found that CD10 and GPR77 were consistently upregulated in the CAFs from patients with chemoresistant cancer.

In cell culture and mouse xenograft models, CD10 ${ }^{+}$GPR77 ${ }^{+}$ CAFs from clinical samples were chemoresistant themselves and rendered co-cultured breast or lung cancer cells similarly chemoresistant. In patient-derived xenograft (PDX) models, the presence of $\mathrm{CD}{ }^{+} \mathrm{GPR} 7^{+} \mathrm{CAF}$ was associated with successful engraftment.

Notably, the number of tumour cells expressing aldehyde dehydrogenase 1 (ALDH1), a marker of CSCs, correlated with the number of $\mathrm{CD} 10^{+} \mathrm{GPR} 77^{+}$ CAFs in breast biopsy samples, and the CAFs physically surrounded these $\mathrm{ALDH}^{+}$cells. Indeed, co-injection of $\mathrm{CD} 10^{+} \mathrm{GPR} 77^{+}$ CAFs isolated from breast or lung cancers increased the proportion of MCF-7 or A549 cells that were positive for ALDH1 in serial transplantation studies, suggesting that $\mathrm{CD} 10^{+} \mathrm{GPR} 77^{+} \mathrm{CAFs}$ provide a niche for CSC survival.

A gene-set enrichment analysis showed that nuclear factor (NF)-KB target genes, including interleukin (IL)-6 and IL-8, were upregulated in CAFs from chemoresistant samples relative to chemosensitive samples. Additional findings in cell culture support a model in which GPR77 engagement by its ligand, C5a, promotes NF- $\mathrm{KB}$ activation, which increases secretion of IL-6 and IL-8. These cytokines promote CSC and CAF survival.

Consistent with this model, intravenous administration of an anti-GPR77 neutralizing antibody had antitumour effects. The antibody reduced PDX establishment if administered during implantation. Administration of the anti-GPR77 antibody after implantation increased the sensitivity of these tumours to docetaxel; the combined therapy reduced tumour growth and increased apoptosis of both the tumour cells and the CAFs.

Next, Song says he plans to explore the effectiveness and feasibility of dual-targeted therapy, to see whether targeting both CD10 and GPR77 is more effective than the anti-GPR77 antibody alone.

Megan Cully

ORIGINAL ARTICLE Su, S. et al. CD $10^{+} \mathrm{GPR} 77^{+}$ Cancer-associated fibroblasts promote cancer formation and chemoresistance by sustaining cancer stemness. Cell 172, 841-856 (2018) 